\title{
OPTIMIZACIÓN DE LA RUGOSIDAD Y LA DUREZA DEL ACERO 12L-14 EMPLEANDO LA METODOLOGÍA DE SUPERFICIE DE RESPUESTA
}

\author{
Wilson Arenas, Oscar Martínez, Marylin Martínez \\ Ricardo Otero-Caicedo, Martha Manrique* \\ Pontificia Universidad Javeriana, Bogotá D.C., Colombia
}

Recibido: 27 de noviembre del 2018 / Aprobado: 16 de mayo del 2019

doi: 10.26439/ing.ind2019.n037.4546

RESUMEN: Este estudio empleó la técnica de superficie de respuesta para optimizar la dureza y rugosidad del acero 12L-14 a partir del tiempo y la temperatura de austenizado. La aplicación multiobjetivo se realizó a partir de un escalamiento con la función de deseabilidad y ponderación, obtenida con la estrategia del proceso analítico jerárquico AHP. Los resultados muestran que la máxima dureza al someter el material a una temperatura de $846,49^{\circ} \mathrm{C}$ durante 48,61 minutos.

Palabras clave: acero 12L-14 / metodología de superficie de respuesta / optimización multiobjetivo / tratamiento térmico / acero-tratamiento térmico

\section{ROUGHNESS AND HARDNESS OPTIMIZATION \\ OF 12L-14 STEEL USING THE RESPONSE SURFACE METHODOLOGY}

ABSTRACT: This research used the response surface technique to optimize $12 \mathrm{~L}-14$ steel hardness and roughness based on the austenitizing time and temperature. The multiobjective problem was managed by scaling the desirability and weighting function using the analytical hierarchical process (AHP). The results show that the maximum hardness is obtained at $846.9^{\circ} \mathrm{C}$ for 48.61 minutes.

Keywords: 12L-14 steel / response surface methodology / multi-objective optimization / heat treatment / steel heat treatment

\footnotetext{
*warenas@javeriana.edu.co, omartinezb@javeriana.edu.co, marylin.martinez@javeriana.edu.co,
} otero@javeriana.edu.co, smanriq@javeriana.edu.co 


\section{INTRODUCCIÓN}

El sector industrial es un campo de constante cambio donde nuevos y numerosos retos exigen a las empresas contar con las mejores herramientas para suplir las demandas de la industria y sus clientes. Entre los puntos de interés más sobresalientes en el área de investigación industrial se encuentra el tratamiento de materiales orientados a la elaboración de piezas como bujías, tuercas, tornillos y partes automotrices, entre otras. Estas piezas deben cumplir altos estándares de resistencia con el fin de minimizar las pérdidas en producción debido a los tiempos improductivos por mantenimiento de los dispositivos. En ese orden de ideas es necesario que la tecnología empleada para fabricar estas piezas sea cada vez mejor, evitando el aumento de costos y al mismo tiempo asegurando la solución de las necesidades de producción y alta calidad de los productos.

En Colombia, el acero, cuya constitución está basada en hierro y carbono, es uno de los materiales más empleados para el diseño de herramientas industriales. El acero 12L-14 es uno de los más usados en la industria automotriz para la fabricación de piezas como tornillos, pernos y tuercas. La alta maquinabilidad de este acero, debido a su alto contenido en plomo y sulfuro de manganeso (tabla 1.) distribuidos de manera equitativa, hace que el material sea de fácil mecanizado (SAE 12L-14). Las especificaciones técnicas de este material se encuentran descritas en la hoja de especificaciones dada por el fabricante; sin embargo, para confiar en estos valores se deben conocer las propiedades que influyen dentro de su proceso de elaboración.

Tabla 1

Composición química del acero 12L-14

\begin{tabular}{ccccc}
\hline$\% \mathrm{C}$ & $\% \mathrm{Mn}$ & $\% \mathrm{P}$ & $\% \mathrm{~S}$ & $\% \mathrm{~Pb}$ \\
\hline 0,15 máx. & $0,85-1,15$ & 0,04 & $0,26-0,35$ & $0,15-0,35$ \\
\hline
\end{tabular}

Fuente: Paredes, 2016

Con el fin de mejorar algunas propiedades del material, como la dureza y la rugosidad, el acero se suele someter a tratamientos térmicos. Por ejemplo, la respuesta del material ante tratamientos térmicos como el temple, varía en cada compuesto produciendo diferentes resultados en la estructura final que no tiende a seguir un comportamiento como se describe en algunas hojas de especificaciones. Por este motivo es natural que las propiedades mecánicas como la dureza y la resistencia a la torsión tengan variaciones significativas entre tratamientos. Las pruebas aplicadas en el proceso de caracterización del material se realizan de manera idea, es decir, su respuesta por lo general varía al someterse a tratamientos reales de ingeniería (Lazcano-Ugalde, Méndez-Macías y Medina-Mendoza, 2016). 
Uno de los estudios más importantes es el análisis del tratamiento térmico de temple de materiales, ya que este incide directamente en la estructura resultante de la aleación tratada, influyendo en su dureza, elasticidad y rugosidad. Este proceso está compuesto por dos fases fundamentales:

1) Calentar el material hasta una temperatura mayor a la crítica para austenizarlo y mantenerlo en esta fase por un tiempo.

2) Realizar el enfriamiento rápido del material (figura 1) (Pérez Ruiz, Galeano Perilla y Negrín Hernández, 2016).

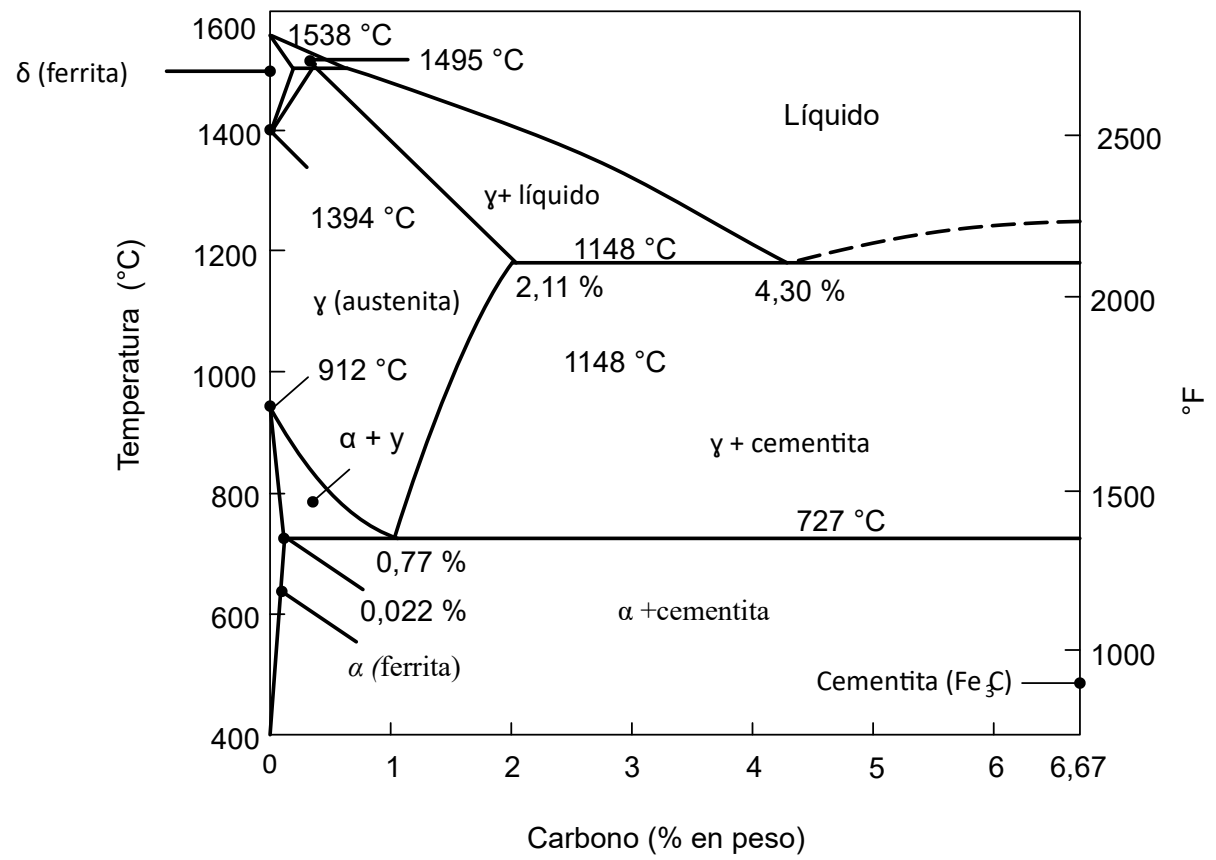

Figura 1. Diagrama de fase hierro-carburo de hierro

Fuente: Kalpakjian y Schmid, 2008

Esta última fase determina la estructura resultante y por consecuencia otorga las propiedades de dureza al material tratado. Caballero y Figueroa (1999) afirman que no existe un método ideal que favorezca la reducción de la temperatura durante el proceso de temple. Sin embargo, hay distintos medios de enfriamiento en el temple de los aceros que pueden tener un efecto cercano al deseado, tales como sumergir el material en agua, soluciones salinas, plomo, aceites, gases y sales fundidas (Pérez Ruiz, Galeano Perilla y Negrín Hernández, 2016). En ese orden de ideas, el tratamiento térmico es muy importante 
en materiales como el acero 12-L14 e influye directamente en su desempeño, especialmente en procesos en que la temperatura es parte fundamental del forjado de las piezas.

Una de las propiedades que más varía en los materiales frente a tratamientos de temple es la dureza. Estudios realizados por Gresesqui-Lobaina, Rodríguez-González y Fernández-Columbié (2017) afirman que al someter a templado materiales ricos en cromo, como el acero 70XL, es posible aumentar el valor de su dureza haciéndolo más resistente. Otro estudio desarrollado por Pérez Ruiz, Galeano Perilla y Negrín Hernández (2016) concluye que el uso de refrigerante de radiador en el proceso de enfriamiento posterior al sometimiento térmico ayuda a que la dureza de aceros ricos en manganeso, como el acero 70XL, el acero AISI 1045 y el acero 12-L14, aumente considerablemente. Con estos estudios se puede deducir que es necesario analizar la influencia de los tratamientos térmicos puntualmente sobre el acero 12L14 en virtud de determinar sus variaciones mecánicas particulares debidas al tratamiento.

Otro parámetro que cambia debido a los tratamientos de temple es la rugosidad. Con el fin de evaluar si una pieza cuenta con la calidad adecuada se debe determinar el valor de la rugosidad superficial (Morales Tamayo, Pérez Rodríguez, Zambrano Robledo, Ávila Rondón y Zamora Hernández, 2014), es decir, las irregularidades ondulatorias en la capa más externa de la superficie de la pieza. Cuando la superficie del acero no tiene un acabado óptimo, el costo de producción aumenta debido a que los equipos que realizan el corte del material deben aumentar su esfuerzo reduciendo su vida útil (Gómez Barrantes y Romero Pinzón, 2017). Del mismo modo, cuando se disminuye la rugosidad de un material es posible aumentar su resistencia debido a que se minimizan los puntos de riesgo que generan grietas de ruptura, evitando así la fractura del acero. De acuerdo con Gómez Barrantes y Romero Pinzón (2017), analizar la rugosidad del material no solo es importante para mejorar las propiedades mecánicas, sino que también contribuye a la elaboración de materiales estéticamente más aceptables y con una vida útil mayor.

Para poder analizar la influencia que tienen ciertos factores sobre una variable de respuesta, es importante aplicar modelos estadísticos que ayuden a determinar cómo variar las condiciones normales de un proceso para encontrar cambios en la respuesta y concluir cuáles de dichas variaciones mejoran o no el proceso. Según De Almeida et al. (2018), emplear estrategias como el diseño de experimentos (DOE) minimiza los costos debido a que esta técnica estadística puede modelar y optimizar experimentos. El DOE cuenta con distintas técnicas que ayudan a plantear y analizar el procedimiento que se debe seguir para llegar a conclusiones objetivas sin sesgo, controlando factores externos que pueden afectar la prueba y permitiendo la selección de los factores que afectan la variable de respuesta con mayor relevancia.

En complemento, para determinar cuál es el mejor tratamiento posible, se emplea la metodología de superficie de respuesta (RSM por sus siglas en inglés). El objetivo es 
diseñar un experimento que proporcione valores razonables de la variable de respuesta $\mathrm{y}$, posteriormente determinar el modelo matemático que mejor se ajusta a los datos obtenidos, es decir, establecer los valores de los factores que optimizan (minimizan o maximizan) el valor de la variable de respuesta. Como definición, la RSM es una herramienta que admite el uso de DOE, técnicas de modelación del análisis de regresión y técnicas matemáticas de optimización, cuya ventaja es que para determinar varios parámetros y la interacción entre ellos emplea un número de pruebas reducido, permitiendo que el desarrollo del experimento y la toma de datos sean más eficientes (Gordillo, Rodríguez y Mejía, 2014; Yaguas, 2017).

Para encontrar el mejor tratamiento posible es necesario analizar las variables de respuesta de manera conjunta y no solo hacer un análisis de estas de manera separada. Esto implica la necesidad de incluir técnicas de optimización multiobjetivo. Existen distintos métodos que han sido aplicados por diferentes autores en sus estudios y generalmente son sencillos de implementar en un diseño experimental. De acuerdo con Raissi y Eslami Farsani (2009), entre estas alternativas están: dar una ponderación a cada variable de respuesta para crear una única función objetivo; diseñar un algoritmo específico para cada problema que encuentre las soluciones óptimas del sistema, y emplear el criterio de error cuadrático medio (MSE por sus siglas en inglés) cuando los datos provienen de una matriz combinada para crear un algoritmo basado en cruce aritmético, entre otras alternativas.

De acuerdo con lo anteriormente descrito, el propósito de este trabajo fue evaluar las características de dureza y rugosidad en una muestra de probetas de acero 12L-14 antes y después del temple. Con el fin de unificar las dos variables de respuesta fueron empleadas dos etapas:

1. Normalizar las variables de respuesta de acuerdo con una función de deseabilidad (Larger-The-Best), dando lugar a condiciones de operación que proporcionan valores de respuesta más deseables (Costa, Lourenço y Pereira, 2011).

2. Implementar el proceso de análisis jerárquico (AHP por sus siglas en inglés) obteniendo las prioridades de cada variable con base en la experiencia y conocimiento de un experto.

Con ello, fundamentado el experimento en la metodología de superficie de respuesta, se determinó el tratamiento óptimo para mejorar las propiedades del material y, de esta manera, contribuir con la elaboración de piezas de mejor calidad, que soporten altas cargas manteniendo un bajo costo de fabricación e incrementando las ganancias a largo plazo. 


\section{ANTECEDENTES}

El DOE se ha convertido en una de las herramientas más eficaces para el análisis de elementos en los que influyen uno o más factores, afectando la variable de respuesta. Según Nekouei et al. (2016), los métodos modernos que se utilizan en el diseño de experimentos permiten analizar el efecto mutuo de dos o más parámetros que intervienen en la estructura resultante del material, promoviendo su homogenización química. Un material distribuido químicamente de manera igualitaria favorece la creación de piezas duraderas y con condiciones aptas para procesos orgánicos. Hasta la actualidad se han investigado procesos para mejorar las propiedades mecánicas de materiales que son usados en soporte de elementos biológicos, específicamente en procesos de osteosíntesis, buscando soportar las características físicas del ser humano sin dejar de lado el factor de apoyo necesario para el crecimiento y anclaje de las estructuras del cuerpo (Serra et al., 2017).

Por otro lado, para comprender mejor el comportamiento de la variable de salida en diseño de experimentos, se han implementado metodologías que permiten realizar un mejor análisis, más objetivo y con bajo sesgo en los resultados. Un ejemplo son los diseños experimentales factoriales que permiten realizar una selección de factores mayormente relevantes que influyen en la variable de respuesta. En la industria frecuentemente se usa RSM como herramienta para optimizar los parámetros de entrada de un proceso (Saba y Raygan, 2017). De esta manera, RSM se ha utilizado para determinar los mejores tratamientos sobre procesos de modificación de materiales tales como procesos de aleación, forjado, distribución química, temple, resistencia, caracterización, optimización, entre otras (Mohammed, Achara y Liew, 2018; Srivastava, Maheshwari, Kundra y Rathee, 2017; Senthilkumar, Tamaizharasan y Gobikannan, 2014).

El uso de RSM en aceros se ha orientado principalmente a determinar los mejores tratamientos para mejorar sus propiedades mecánicas, tales como dureza, rugosidad y elasticidad, con el fin de darle mayor durabilidad a las piezas forjadas con estos materiales y, finalmente, impactar en los costos de producción de las grandes empresas manufactureras (Saba y Raygan, 2017; Senthilkumar, Tamaizharasan y Gobikannan, 2014; Bagaber y Yusoff, 2017; Bagaber y Yusoff, 2018; Parida, Routara y Bhuyan, 2015). En la actualidad, el titanio se usa debido a su resistencia a la corrosión, su alta resistencia al peso y la resistencia a la fatiga. Algunos estudios con este material han sido orientados a determinar la velocidad de corte, la velocidad de avance, el desgaste del flanco de la herramienta y las vibraciones de la herramienta de corte en la rugosidad de superficie del material. En este caso, la RSM ha contribuido a determinar los valores óptimos de rugosidad media y rugosidad total en que la pieza resiste de la mejor manera las tensiones inducidas (D'Mello y Pai, 2018). 
Tratándose del área ingenieril, en diversas ocasiones se genera la necesidad de realizar la optimización de variables de respuesta de manera conjunta o multiobjetivo. Con ello se contemplan diferentes variables de respuesta, otorgándoles una jerarquía subjetiva para realizar un análisis simple y determinar la mejor combinación de tratamientos para una variable transformada. En ese orden de ideas, es muy común el uso de RSM junto con la optimización multiobjetivo, puesto que es necesario determinar las mejores condiciones que permitan lograr los objetivos propuestos (Ye, Yang y Liu, 2018; Koohestanian, Samimi, Mohebbi-Kalhori y Sadeghi, 2017). No obstante, puede que la optimización de un objetivo conlleve al empeoramiento del enésimo objetivo. Como ejemplo, en el sector petrolero es ampliamente utilizada para mejorar los procesos de refinamiento de combustibles y así optimizar el rendimiento de determinados motores (Koohestanian, Samimi, Mohebbi-Kalhori y Sadeghi, 2017; Awad et al., 2017).

Para llevar a cabo la optimización multiobjetivo es necesario implementar una función de normalización para garantizar que las n-respuestas se encuentren bajo un mismo rango y no tengan mayor relevancia las de magnitudes más grandes. Una de las alternativas ha sido generar las ecuaciones de la superficie de respuesta para cada una de las variables de manera independiente y emplear técnicas de optimización multiobjetivo. Sin embargo, se debe tener en cuenta que lo anterior funciona solo si las variables de respuesta son independientes. En la mayoría de los casos esto no es así y por este motivo se utilizan combinaciones lineales de variables normalizadas o métodos de funciones de deseabilidad. La función de deseabilidad permite normalizar diferentes variables de respuesta en virtud de acotar la respuesta y generar propiedades estadísticas óptimas deseables (Aouici, Bouchelaghem, Yallese, Elbah y Fnides, 2014). En la tabla 2 se muestra un resumen de los estudios más importantes que se han realizado con aceros.

Tabla 2

Antecedentes del proceso de templado y RSM en materiales

\begin{tabular}{lllll}
\hline \multicolumn{1}{c}{ Referencia } & \multicolumn{1}{c}{ Factores } & \multicolumn{1}{c}{ Técnica } & $\begin{array}{c}\text { Población de } \\
\text { medición }\end{array}$ & \multicolumn{1}{c}{$\begin{array}{c}\text { Variable } \\
\text { de respuesta }\end{array}$} \\
\hline $\begin{array}{l}\text { Sáenz et. al., } \\
2007\end{array}$ & $\begin{array}{l}\text { Tiempo de } \\
\text { envejecimiento }\end{array}$ & $\begin{array}{l}\text { Microscopía óptica } \\
\text { y microscopía } \\
\text { electrónica de } \\
\text { barrido (MEB) }\end{array}$ & $\begin{array}{l}\text { Acero inoxidable } \\
\text { dúplex SAF 2507 }\end{array}$ & $\begin{array}{l}\text { Tracción, impacto } \\
\text { y dureza }\end{array}$ \\
\hline $\begin{array}{l}\text { Morales, } \\
\text { Hormaza, y } \\
\text { Méndez, 2009 }\end{array}$ & $\begin{array}{l}\text { Temperatura } \\
\text { desestabilización }\end{array}$ & $\begin{array}{l}\text { Microscopía } \\
\text { óptica con análisis } \\
\text { estadístico } \\
\text { descriptivo }\end{array}$ & $\begin{array}{l}\text { Aleación del tipo } \\
\text { 26Cr.5Mo }\end{array}$ & Microestructura \\
\hline $\begin{array}{l}\text { Posada Ramírez, dureza } \\
2009\end{array}$ & $\begin{array}{l}\text { Velocidad de } \\
\text { corte y avance } \\
\text { del corte }\end{array}$ & Modelo bifactorial & Acero AISI12L14 & Dureza \\
\hline
\end{tabular}


(continuación)

\begin{tabular}{|c|c|c|c|c|}
\hline $\begin{array}{l}\text { Morales Villamil, } \\
2009\end{array}$ & $\begin{array}{l}\text { Profundidad de } \\
\text { corte, avance y } \\
\text { revoluciones del } \\
\text { husillo }\end{array}$ & $\begin{array}{l}\text { RSM, regresión } \\
\text { estadística, diseño } \\
\text { Taguchi }\end{array}$ & $\begin{array}{l}\text { Aluminio } \\
\text { SAE } 1060 \text {, bronce } \\
\text { SAE } 40 \text { y acero } \\
\text { SAE } 12 \text { L } 14\end{array}$ & Mecanizado \\
\hline $\begin{array}{l}\text { Robledo y } \\
\text { Cárdenas, } 2011\end{array}$ & $\begin{array}{l}\text { Temperatura y } \\
\text { tiempo de temple } \\
\text { y revenido }\end{array}$ & $\begin{array}{l}\text { OFAT: mover un } \\
\text { factor a la vez }\end{array}$ & $\begin{array}{l}\text { Acero } \\
\text { AISISAE } 1045\end{array}$ & $\begin{array}{l}\text { Microestructura y } \\
\text { dureza }\end{array}$ \\
\hline $\begin{array}{l}\text { Artigas et. al., } \\
2012\end{array}$ & $\begin{array}{l}\text { Temperatura } \\
\text { austenizado }\end{array}$ & $\begin{array}{l}\text { Análisis } \\
\text { metalográficos } \\
\text { y ensayos } \\
\text { mecánicos. }\end{array}$ & $\begin{array}{l}\text { Acero al CMnSi } \\
\text { AWS } 70 S 6\end{array}$ & Fluencia, UTS y dureza \\
\hline $\begin{array}{l}\text { Pérez, Olaya y } \\
\text { Arenas, } 2013\end{array}$ & $\begin{array}{l}\text { Temperatura de } \\
\text { austenizado y } \\
\text { revenido }\end{array}$ & $\begin{array}{l}\text { Análisis estadístico } \\
\text { descriptivo }\end{array}$ & Acero AISI $5160 \mathrm{H}$ & $\begin{array}{l}\text { Microestructura, } \\
\text { dureza, tenacidad y } \\
\text { tensión }\end{array}$ \\
\hline $\begin{array}{l}\text { Franco y } \\
\text { Harrinson Paz, } \\
2004\end{array}$ & $\begin{array}{l}\text { Tiempo de } \\
\text { austenizado }\end{array}$ & $\begin{array}{l}\text { Análisis de } \\
\text { variables con } \\
\text { estadística } \\
\text { descriptiva }\end{array}$ & $\begin{array}{l}\text { Aceros AISI } 1018 \\
\text { y } 1025\end{array}$ & Dureza \\
\hline $\begin{array}{l}\text { Aouici, } \\
\text { Bouchelaghem, } \\
\text { Yallese, Elbah y } \\
\text { Fnides, } 2014\end{array}$ & $\begin{array}{l}\text { Velocidad } \\
\text { de corte y } \\
\text { alimentación, y } \\
\text { profundidad de } \\
\text { corte }\end{array}$ & $\begin{array}{l}\text { RSM, función de } \\
\text { deseabilidad }\end{array}$ & Acero AISI D3 & $\begin{array}{l}\text { Rugosidad de la } \\
\text { superficie, fuerza de } \\
\text { corte, fuerza de corte } \\
\text { específica y potencia }\end{array}$ \\
\hline $\begin{array}{l}\text { Silva Urueña } \\
\text { y Castañeda } \\
\text { Vargas, } 2015\end{array}$ & Temperatura & $\begin{array}{l}\text { Comparación } \\
\text { estadística entre } \\
\text { datos teóricos y } \\
\text { experimentales }\end{array}$ & Acero SAE 1045 & $\begin{array}{l}\text { Tensión, impacto y } \\
\text { dureza }\end{array}$ \\
\hline Pérez et. al., 2015 & $\begin{array}{l}\text { Velocidad de } \\
\text { corte }\end{array}$ & $\begin{array}{l}\text { RSM y redes } \\
\text { neuronales (NN) }\end{array}$ & $\begin{array}{l}\text { Acero cold rolled } \\
1018\end{array}$ & Rugosidad superficial \\
\hline $\begin{array}{l}\text { Ariza, Martorano } \\
\text { y Tschiptschin, } \\
2015\end{array}$ & $\begin{array}{l}\text { Temperatura de } \\
\text { austenizado }\end{array}$ & $\begin{array}{l}\text { Método de } \\
\text { elementos finitos }\end{array}$ & $\begin{array}{l}\text { Acero AISI/SAE } \\
4340\end{array}$ & Tensiones residuales \\
\hline $\begin{array}{l}\text { Soria-Aguilar } \\
\text { et. al., } 2015\end{array}$ & $\begin{array}{l}\text { Temperatura } \\
\text { y tiempo de } \\
\text { austenizado y } \\
\text { revenido }\end{array}$ & $\begin{array}{l}\text { Diseño Taguchi. } \\
\text { ANOVA del arreglo } \\
\text { Taguchi }\end{array}$ & $\begin{array}{l}\text { Acero API 5CT } \\
\text { J55 }\end{array}$ & Dureza y tensión \\
\hline $\begin{array}{l}\text { Parida, Routara y } \\
\text { Bhuyan, } 2015\end{array}$ & $\begin{array}{l}\text { Velocidad } \\
\text { del husillo, } \\
\text { alimentación y } \\
\text { diámetro de la } \\
\text { broca }\end{array}$ & $\begin{array}{l}\text { RSM, diseño } \\
\text { Taguchi ortogonal, } \\
\text { ANOVA }\end{array}$ & Compuesto GFRP & Rugosidad superficial \\
\hline
\end{tabular}


(continuación)

\begin{tabular}{|c|c|c|c|c|}
\hline $\begin{array}{l}\text { González } \\
\text { Guevara, } \\
\text { Manrique } \\
\text { Torres y Quiroga } \\
\text { Sanchez, } 2016\end{array}$ & $\begin{array}{l}\text { Temperatura } \\
\text { austenizado }\end{array}$ & ANOVA & $\begin{array}{l}\text { Acero K } 340 \text {, AISI } \\
\text { A2 y AISI D6 }\end{array}$ & Dureza \\
\hline $\begin{array}{l}\text { Nekouei et al., } \\
2016\end{array}$ & $\begin{array}{l}\text { Temperatura } \\
\text { y tiempo de } \\
\text { austenizado }\end{array}$ & RSM & Acero $30 \mathrm{CrMnSi}$ & $\begin{array}{l}\text { Propiedades } \\
\text { mecánicas }\end{array}$ \\
\hline $\begin{array}{l}\text { Rodríguez- } \\
\text { Baracaldo, } \\
\text { Arroyo-Osorio y } \\
\text { Parra-Rodríguez, } \\
2016\end{array}$ & $\begin{array}{l}\text { Temperatura de } \\
\text { revenido }\end{array}$ & MEB & Acero DP580 & $\begin{array}{l}\text { Microestructura, } \\
\text { dureza y tracción }\end{array}$ \\
\hline $\begin{array}{l}\text { Lazcano-Ugalde, } \\
\text { Méndez-Macías } \\
\text { y Medina- } \\
\text { Mendoza, } 2016\end{array}$ & $\begin{array}{l}\text { Temperatura y } \\
\text { tensión }\end{array}$ & $\begin{array}{l}\text { Análisis estadístico } \\
\text { descriptivo }\end{array}$ & Acero AISI 12L-14 & $\begin{array}{l}\text { Resistencia a la } \\
\text { tensión, límite } \\
\text { elástico, elongación y } \\
\text { reducción de área }\end{array}$ \\
\hline $\begin{array}{l}\text { Gómez } \\
\text { Barrantes y } \\
\text { Romero Pinzón, } \\
2017\end{array}$ & $\begin{array}{l}\text { Velocidad de } \\
\text { corte, del husillo } \\
\text { de avance y } \\
\text { profundidad }\end{array}$ & RSM & AISI/SAE 1045 & Rugosidad superficial \\
\hline $\begin{array}{l}\text { Gresesqui- } \\
\text { Lobaina, } \\
\text { Rodríguez- } \\
\text { González y } \\
\text { Fernández- } \\
\text { Columbié, } 2017\end{array}$ & $\begin{array}{l}\text { Tratamiento } \\
\text { térmico y } \\
\text { revenido }\end{array}$ & $\begin{array}{l}\text { Análisis estadístico } \\
\text { descriptivo }\end{array}$ & Acero AISI 4140 & Dureza y peso \\
\hline Serra, et al., 2017 & $\begin{array}{l}\text { Temperatura } \\
\text { y tiempo de } \\
\text { austenizado }\end{array}$ & $\begin{array}{l}\text { Análisis de } \\
\text { microestructura } \\
\text { por observación y } \\
\text { análisis estadístico } \\
\text { descriptivo }\end{array}$ & $\begin{array}{l}\text { Acero AISI 304L y } \\
\text { AISI 316L }\end{array}$ & $\begin{array}{l}\text { Microestructura, } \\
\text { dureza, tenacidad y } \\
\text { tensión }\end{array}$ \\
\hline $\begin{array}{l}\text { Saba y Raygan, } \\
2017\end{array}$ & $\begin{array}{l}\text { Duración de } \\
\text { la molienda, } \\
\text { tamaño de las } \\
\text { partículas de } \\
\text { polvo fino y } \\
\text { grueso y sustrato } \\
\text { endurecido y } \\
\text { recibido }\end{array}$ & $\begin{array}{l}\text { Difracción de rayos } \\
\text { X y microscopía } \\
\text { electrónica de } \\
\text { barrido (SEM) }\end{array}$ & $\begin{array}{l}\text { Sustrato de acero } \\
\text { AISI D2 }\end{array}$ & $\begin{array}{l}\text { Espesor del } \\
\text { recubrimiento, la } \\
\text { microdureza y el } \\
\text { número de dureza al } \\
\text { rayado }\end{array}$ \\
\hline
\end{tabular}


(continuación)

\begin{tabular}{lllll}
\hline D'Mello, Pai y & Velocidad de & Redes neuronales & Ti-6Al-4V & Rugosidad aparente y \\
Puneet, 2018 & corte, velocidad & artificiales: & & Multi Layer \\
& de avance, & & \\
& desgaste de la & Perceptron, Radial & & \\
& herramienta y & Basis Function & & \\
& vibraciones de la & Neural Network & & \\
& herramienta de & y Summation & & \\
& corte & Wavelet- Extreme & & \\
& & Learning Machine & & \\
\hline Lario, Castaño, & Temperatura & Microscopia & Nanotubos TiO 2 & Rugosidad superficial \\
Viera, Segovia y & y tiempo de & electrónica de & & \\
Amigo, 2018 & revenido & barrido de emisión & & \\
& & de campo & & \\
\hline
\end{tabular}

Elaboración propia

En este trabajo se va a analizar la influencia del tratamiento térmico de temple sobre la dureza y la rugosidad del acero 12L-14, teniendo como referencia los métodos de análisis estadísticos empleados en la literatura, como por ejemplo RSM, función multiobjetivo y AHP, con el fin de obtener los límites de temperatura bajo los cuales el acero 12L-14 presenta máxima dureza.

\section{METODOLOGÍA}

Para poder realizar este experimento se aplica el desarrollo de etapas mostrado en la figura 2, en donde se evidencian las actividades a realizar.

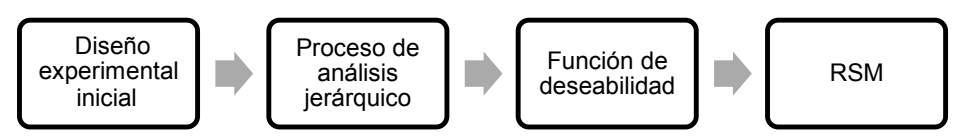

Figura 2. Metodología propuesta

Elaboración propia

\subsection{Diseño experimental inicial}

Para determinar las condiciones de operación basadas en la temperatura y tiempo de austenizado, que maximiza la dureza del acero y afecta la rugosidad del material, se tomó una muestra de 36 probetas de acero 12L-14, en forma de cilindro de $627,4 \mathrm{~mm}^{3}$ (20 $\mathrm{mm}$ de alto por $6,32 \mathrm{~mm}$ de diámetro). Se partió de un diseño experimental $3^{2}$ y posteriormente, con estos resultados, se realizó la superficie de respuesta de primer orden, teniendo como referencia la ecuación de regresión y los respectivos gradientes. 
Para la obtención de los valores máximos y mínimos de temperatura de austenizado se tomó el rango de temperaturas sugeridos por el fabricante siendo este desde 820 hasta $850^{\circ} \mathrm{C}$. El rango de tiempo de austenizado fue determinado por la experiencia y conocimiento al trabajar con el material (considerando el espesor y composición química del acero), siendo este de 30 a 50 minutos.

\subsection{Proceso de análisis jerárquico}

Una de las metodologías propuestas para la solución de problemas multicriterio es el proceso de análisis jerárquico (AHP), el cual evalúa alternativas cuando se tiene más de una variable de respuesta y se fundamenta en la experiencia y el conocimiento de expertos. La ecuación de unificación de variables de respuesta por el método de AHP es:

$$
\varphi=w_{-} D \cdot D+w_{-} R \cdot R
$$

De acuerdo con Saaty (2008), AHP realiza comparaciones entre pares de elementos para establecer las respectivas prioridades frente al objetivo principal. En este orden de ideas y con base en la experiencia se determinaron las variables que son relevantes al momento de realizar tratamientos de temple. De este análisis se concluyó que los criterios relevantes al tratar un material son: costo, funcionalidad, durabilidad y rigidez. Los resultados de las comparaciones por pares se exponen en las tablas 3 y 4 .

Tabla 3

Matriz de comparación de los criterios

\begin{tabular}{lcccc}
\hline \multicolumn{1}{c}{ Criterio } & Costo & Funcionalidad & Durabilidad & Rigidez \\
\hline Costo & 1 & $1 / 5$ & $1 / 6$ & 6 \\
Funcionalidad & 5 & 1 & 1 & 7 \\
Durabilidad & 6 & 1 & 1 & 7 \\
Rigidez & $1 / 6$ & $1 / 7$ & $1 / 7$ & 1 \\
\hline
\end{tabular}

Elaboración propia

Tabla 4

Matriz de prioridad de los criterios

\begin{tabular}{cccccc}
\hline $\begin{array}{c}\text { Prioridad de las } \\
\text { alternativas }\end{array}$ & Costo & Funcionalidad & Durabilidad & Rigidez & Resultado \\
\hline Dureza & 0,0204 & 0,2018 & 0,3455 & 0,0211 & 0,5888 \\
Rugosidad & 0,1019 & 0,2018 & 0,0864 & 0,0211 & 0,4111 \\
\hline
\end{tabular}

Elaboración propia 


\subsection{Función de deseabilidad}

Ya que las variables de respuesta se encuentran bajo diferentes unidades y oscilan en diferentes rangos, es injusto realizar la unificación de las variables sin haber realizado una estandarización previa. Con esto se busca que ambas variables de respuesta oscilen entre 0 y 1 y así, que los pesos $W_{D}$ y $W_{R}$ otorgados por el AHP sean los únicos descriptores de la relación entre ellas.

Con base en las normas ISO 4287, DIN 4762, DIN 4768 (Surface Roughness Testing) y en los datos experimentales de rugosidad aparente luego de la prueba de temple, el rango de esta variable para métodos de mecanizado individuales está dado entre los grados de rugosidad 0 y 2 , es decir, entre 0,0125 y 0,05 .

Con respecto a la dureza, el acero 12L-14 no responde a tratamientos de temple convencionales, por lo que no es posible determinarla mediante temple convencional. Ante esto, los rangos de la variable fueron determinados por la experiencia y conocimiento del experto. De esta manera, la dureza del acero 12L-14 después de un proceso de temple y bajo las condiciones de tiempo prestablecidas se encuentra entre 195 y 290 Vickers.

A partir de los mínimos y máximos de las variables en cuestión se emplea una función de deseabilidad (Larger-The-Best), dando lugar a condiciones de operación que proporcionan valores de respuesta más deseables (Costa, Lourenço y Pereira, 2011), dada por:

$$
d=\left(\frac{\hat{y}-L}{U-L}\right), L \leq \hat{y} \leq U
$$

Donde $\hat{y}$ es la variable de respuesta a normalizar y $L$ y $U$ y son el valor mínimo y máximo deseable de la variable de respuesta, respectivamente.

Finalmente, hallada la normalización es posible realizar la unificación de las variables de respuesta reemplazando (I) con los resultados de la tabla 3 , de la siguiente manera:

$$
\varphi=0,5888 \cdot\left(\frac{D-195}{95}\right)+0.4111 \cdot\left(\frac{R-0,0125}{0,0375}\right)
$$

Donde $\varphi=$ es la variable de respuesta normalizada $[0,1]$ dependiente de la dureza y rugosidad aparente, $D$ es la dureza en Vickers y $R$ es la rugosidad aparente en $\mu \mathrm{m}$.

\section{MÉTODOS Y MATERIALES}

Con el fin de recopilar los datos de dureza se siguen los pasos mostrados en la figura 3. Para comprender mejor la manera en la que fue desarrollado, se explica detalladamente cada uno de los apartados a continuación. 


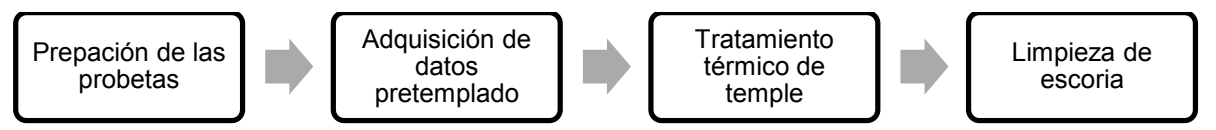

Figura 3. Etapas del desarrollo experimental

Elaboración propia

Para la preparación de las probetas se cortó la varilla de acero 12L-14 para la obtención de 18 probetas. Cada una de estas fue limada por su dorso mediante una lima plana bastarda, hasta obtener una superficie plana. Posteriormente, por medio del MP-2B Grinder Polisher y una lija n. 320 se lijó la superficie obtenida de manera horizontal a 175 revoluciones por minuto y luego se pulieron con una lija n. 600 que giraba con las mismas revoluciones mencionadas.

Con el fin de evitar confusión en las muestras se etiquetaron las probetas de manera aleatoria y se capturaron imágenes de cada una de estas con ayuda del microscopio invertido metalográfico (figura 4). A continuación se procedió con la medición de la rugosidad del material. Para esto se calibró el rugosímetro Mitutoyo SJ210 y se midió dicho parámetro inicial en tres diferentes puntos espaciales para cada una de las muestras; de allí se calculó el valor medio a partir de los valores obtenidos. Luego se obtuvo el valor medio entre las réplicas de las probetas.
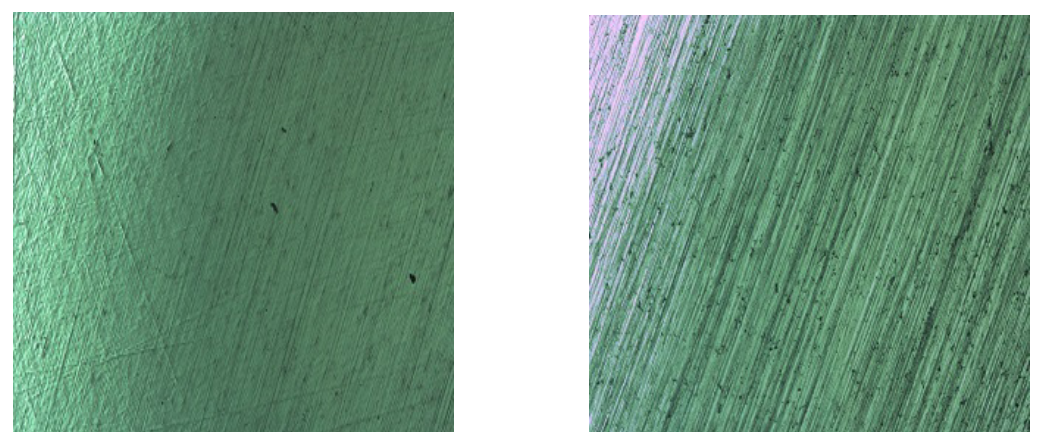

Figura 4. Vista al microscopio de la probeta 1.1 antes del tratamiento térmico (izquierda) y después del tratamiento térmico (derecha)

Elaboración propia

Para la medición de dureza se empleó el microdurómetro HVS1000A (fuerza de $0,981 \mathrm{~N}$ y duración de fijación de 10 segundos). Como primera instancia se calibró el dispositivo y posteriormente se midió la dureza inicial en tres puntos espaciales diferentes para cada una de las probetas, obteniendo el valor medio a partir de las tres mediciones. Finalmente se calculó el valor medio entre las réplicas de las probetas. 
Para el tratamiento térmico se deben introducir las probetas al horno durante un tiempo determinado a una temperatura especifica e invariante. Posteriormente es necesario retirar las probetas del horno y rápidamente bajar la temperatura sumergiéndolas en aceite. Ya que este experimento contempla diferentes mediciones variando los dos factores, es necesario replicar los tratamientos de tal forma que se contemple la realización de todas las combinaciones con el procedimiento previamente descrito. En este caso se utilizó el horno Borel KP 11001 a una temperatura de austenizado de $820{ }^{\circ} \mathrm{C}$ durante treinta minutos. Luego se ubicaron dos nuevas probetas durante cuarenta minutos $y$, finalmente, se introdujeron las últimas dos probetas para esta temperatura durante un tiempo de cincuenta minutos. Esto para cada una de las dos temperaturas restantes.

Por último, por medio de la pulidora industrial se pulieron las probetas nuevamente con una lija n. ${ }^{\circ} 600$ sobre su dorso plano para eliminar las partículas de óxido originadas por el tratamiento térmico. Seguidamente, se limpiaron y se tomaron los datos referentes a dureza y rugosidad en tres puntos espaciales diferentes de las muestras.

\section{METODOLOGÍA DE SUPERFICIE DE RESPUESTA}

A continuación se describen de manera general los pasos realizados para poder obtener una función matemática que permita establecer la relación existente entre las variables de respuesta y los factores de entrada.

Experimento inicial: recolección inicial de datos experimentales con el propósito de estimar el efecto que los factores tienen sobre las variables de respuesta. En este caso se suelen utilizar diseños con baja granularidad de exploración pues solo se desea conocer una estimación lineal de la superficie de respuesta.

Exploración de la región de búsqueda: una vez que se haya estimado una relación lineal, usualmente a partir de una regresión por mínimos cuadrados, se procede a una exploración del espacio de búsqueda, realizando variaciones en los valores de los factores. En primer lugar, con esto se busca optimizar la variable de respuesta con cada una de las variaciones sucesivas de los factores y también tiene como propósito encontrar zonas de curvatura, donde es necesario utilizar diseños factoriales de mayor orden para analizar posibles efectos no lineales. En este caso se agregan puntos axiales que permitan estimar de forma eficiente efectos de curvatura.

Obtención de los valores óptimos de los niveles de los factores: una vez que se haya estimado una ecuación de regresión con componentes polinomiales, se procede a utilizar algoritmos de optimización no lineal que permitan encontrar los valores del factor temperatura y del factor tiempo que maximicen la dureza.

Experimento de verificación: para garantizar que los resultados han sido obtenidos de manera adecuada es necesario realizar una medición experimental adicional en los 
puntos considerados óptimos para garantizar que la dureza sea mayor a las encontraras previamente.

\section{RESULTADOS}

El experimento inicial fue un $3^{k}$ con el fin único de medir el efecto de los factores a partir de un análisis de varianza ANOVA. Si bien en estas situaciones usualmente se utiliza tan solo un diseño $2^{k}$, se contó con la oportunidad de tomar más mediciones con el fin de obtener una mejor estimación de su relación lineal.

Inicialmente se tomaron los valores de dureza y rugosidad cuando las probetas no habían sido sometidas al proceso de temple. Teniendo dichos datos se realizó el tratamiento térmico.

Después de limpiar y lijar las probetas se tomaron nuevamente los datos correspondientes a las variables de respuesta con el fin de analizar su variación respecto a los parámetros que se modificaron durante el tratamiento térmico (tabla 5).

Posteriormente se determinó la ecuación de regresión a partir de mínimos cuadrados.

$$
y=\eta+\varepsilon=f\left(x_{1}, x_{2}\right)+\varepsilon
$$

Donde $\eta$ es la función de rendimiento, que está en función del tiempo de austenizado $\left(x_{1}\right)$ y de la temperatura de austenizado $\left(x_{2}\right)$, y $\varepsilon$ es el error observado en las variables de respuesta.

Después del análisis de varianza se obtuvieron p-values de 0,236, 0,300, 0,099 y 0,158 , referentes al intercepto, tiempo de austenizado, temperatura de austenizado y ecuación de regresión, respectivamente. Esto indica que, usando como referencia un nivel de confianza del $95 \%$, ninguno de los factores evaluados fue significativo estadísticamente. A partir de estos resultados y con el fin de analizar por qué los factores no son significativos, se realizó la regresión para cada variable de respuesta, sin tener presente el proceso de análisis jerárquico ni la función de deseabilidad (tabla 6).

De acuerdo con lo anterior se evidencia que al tener en cuenta la dureza como variable de respuesta, tanto el tiempo como la temperatura de austenizado son significativos. En contraste, cuando la variable de respuesta se basa en la rugosidad, ni el tiempo ni la temperatura de austenizado son significativos. Por ende, cuando se analiza la regresión de la variable de respuesta ponderada a partir de AHP, esta no es significativa. Teniendo en cuenta lo anterior se infiere que para los rangos medidos los factores tiempo y temperatura de austenizado no están relacionados con la rugosidad. 


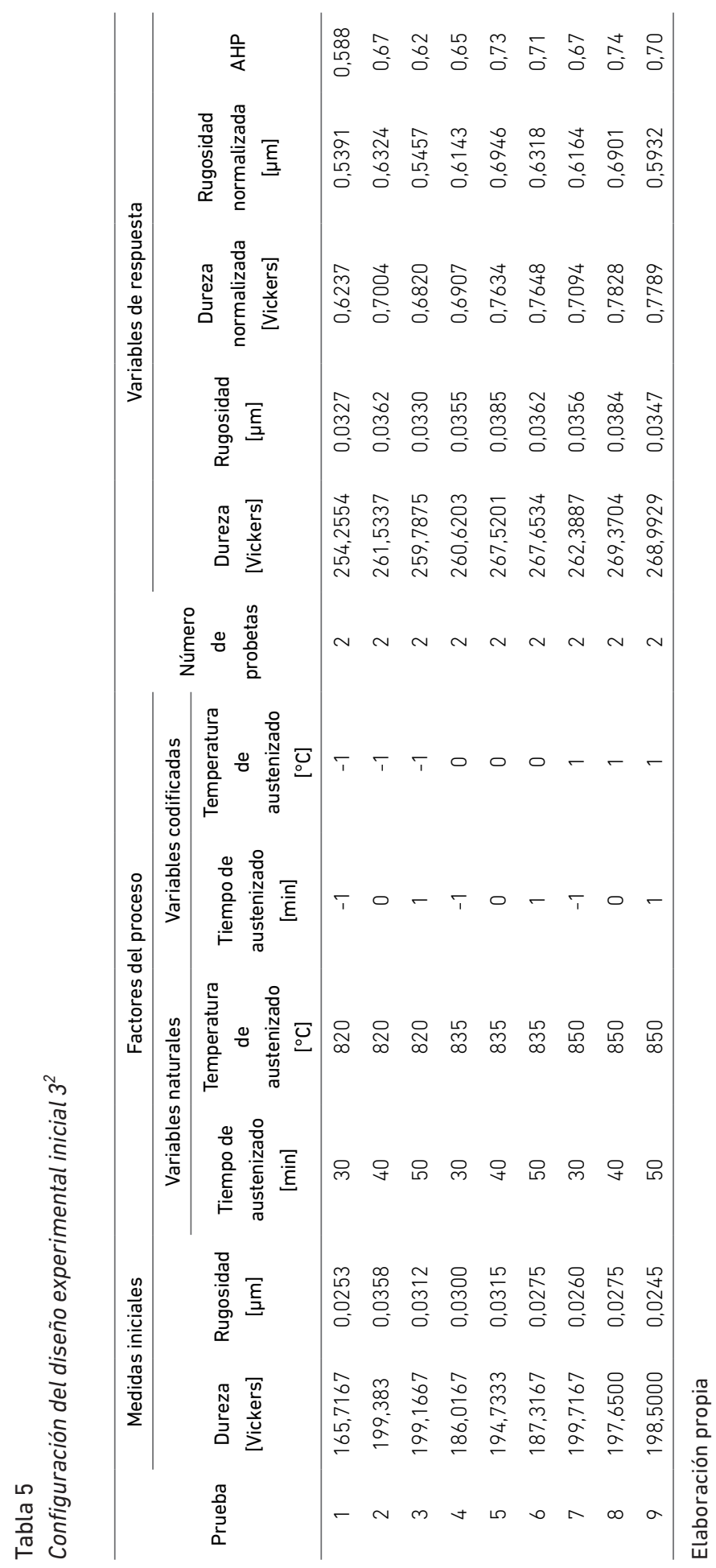


Tabla 6

Resultados de la regresión para cada una de las variables de respuesta

\begin{tabular}{llccccc}
\hline $\begin{array}{l}\text { Variable de } \\
\text { respuesta }\end{array}$ & \multicolumn{1}{c}{ Factor } & Estimado & $\begin{array}{c}\text { Error } \\
\text { estándar }\end{array}$ & Valor T & $\operatorname{Pr}(>|\mathrm{t}|)$ & $\begin{array}{c}p \text {-value } \\
\text { regresión }\end{array}$ \\
\hline Dureza & Intercepto & 41,1471 & 71,0924 & 0,5790 & 0,5838 & \\
& Tiempo de austenizado & 0,3195 & 0,1300 & 2,4590 & 0,0492 & 0,0240 \\
& Temperatura de austenizado & 0,2516 & 0,0851 & 2,9570 & 0,0254 & \\
\hline Rugosidad & Intercepto & $-1,54 \mathrm{E}-02$ & $4,80 \mathrm{E}-02$ & $-0,3210$ & 0,7590 & \\
& Tiempo de austenizado & $1,67 \mathrm{E}-06$ & $8,772-05$ & 0,0190 & 0,9850 & 0,5944 \\
& Temperatura de austenizado & $6,12 \mathrm{E}-05$ & $5,74 \mathrm{E}-05$ & 1,0660 & 0,3280 & \\
\hline Dureza + & Intercepto & $-1,2615$ & 0,9580 & $-1,3170$ & 0,2360 & \\
rugosidad & Tiempo de austenizado & 0,0020 & 0,0018 & 1,1340 & 0,3002 & 0,1588 \\
& Temperatura de austenizado & 0,0022 & 0,0011 & 1,9480 & 0,0993 &
\end{tabular}

Elaboración propia

Por lo tanto, teniendo en cuenta el protocolo del diseño de experimentos realizado, donde el tiempo inicial para las probetas dentro del horno fue corto (menos de diez minutos) y, de acuerdo con la regresión realizada para cada variable por separado, se puede concluir que solo la dureza obtiene valores significativos en tiempo y temperatura. Por este motivo el presente documento se fundamentará sobre la variable dureza. Así se realiza un análisis de la estadística descriptiva de los datos a partir de los valores encontrados. En la figura 5 se pueden ver los diagramas de caja para cada factor.

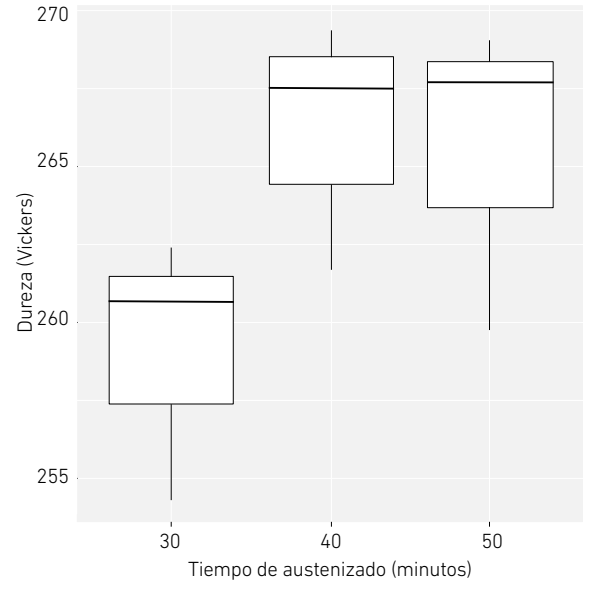

A

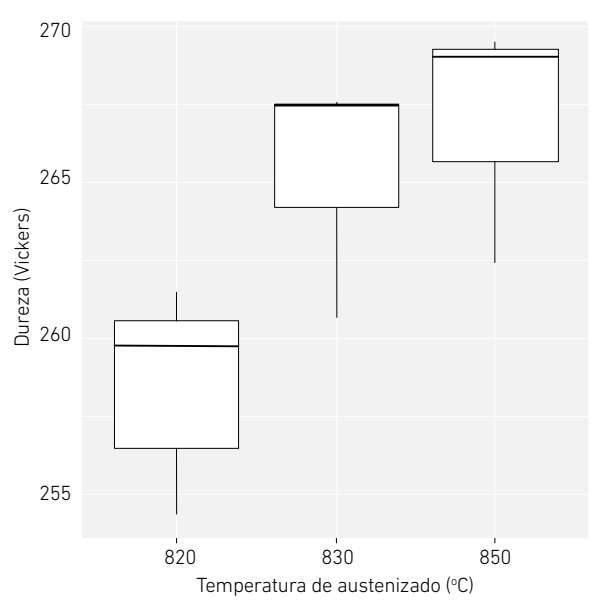

B

Figura 5. Diagrama de cajas de los factores según la variable de respuesta. A: tiempo de austenizado y B: temperatura de austenizado

Elaboración propia 
La normalidad de los residuos fue verificada a través de la prueba de bondad de ajuste de Anderson-Darling ( $p>0.05$ ); la independencia de los residuos fue verificada a través de un gráfico de tendencia y una prueba de rachas $(p>0.05)$. El análisis de varianza se presenta en la tabla 7. Como referencia se usó un nivel de confianza del $95 \%$.

Tabla 7

ANOVA de los datos de dureza postemple

\begin{tabular}{lccccc}
\hline Factor & SC & CM & DF & Valor F & $\operatorname{Pr}(>\mathrm{F})$ \\
\hline Tiempo de austenizado & 91,0183 & 45,5092 & 2 & 172,5794 & 0,0001 \\
\hline Temperatura de austenizado & 118,5689 & 59,2845 & 2 & 224,818 & 0,0001 \\
\hline Residual & 1,0546 & 0,2637 & 4 & & \\
\hline
\end{tabular}

Elaboración propia

Y su ecuación de regresión está dada por:

$$
y=17.218+0,25 x_{1}+0,1968 x_{2}
$$

Según el ANOVA, el valor del coeficiente $R^{2}=99 \%$ muestra el buen ajuste del modelo lineal, indicando que en esta región de exploración inicial no se requieren estimaciones de efectos polinómicos. Para la fase de exploración de la región de búsqueda se implementó el algoritmo de gradiente descendiente. Para definir la variable que guiará el tamaño del paso de la trayectoria se eligió la variable del proceso cuyo coeficiente de regresión absoluto fue mayor. En este sentido, el mayor coeficiente corresponde al factor de tiempo de austenizado, por lo que se determinó que gradiente de $x_{1}$ igual a $\Delta x_{1}=0,25$. Con ello, el gradiente para estuvo dado por $\Delta x_{2}=0,1968$, por lo que estos gradientes en unidades naturales de tiempo y temperatura de austenizado fueron $\Delta \xi_{1}=2,5 \mathrm{~min}$ y $\Delta \xi_{2} \approx 3{ }^{\circ} \mathrm{C}$. Tomando el punto correspondiente a 0,0 , y un tamaño de paso de 1,0 se realizan experimentos individuales adicionales, incrementando las variables en los pasos previamente enunciados (tabla 8 y figura 6).

Se observó que el tercer punto representaba el valor máximo de la trayectoria de experimentación por lo que se actualizó el nuevo punto central $(0,0)$ al punto 47,5, 844 y la región de experimentación para $\xi_{1}$ fue de 45 a 50 minutos y para $\xi_{1}$ fue de 841 a 847 ${ }^{\circ} \mathrm{C}$, obteniendo los datos mostrados en la tabla 9 . En este caso, resulta evidente que cerca de este punto se forma una curvatura, por lo que es necesario generar puntos axiales adicionales que permitan estimarla. En este experimento se generaron los puntos axiales con $\alpha$. 
Tabla 8

Trayectoria de experimentación

\begin{tabular}{|c|c|c|c|c|c|}
\hline & \multicolumn{4}{|c|}{ Variables } & \multirow{3}{*}{ Dureza } \\
\hline & \multicolumn{2}{|c|}{ Codificadas } & \multicolumn{2}{|c|}{ Naturales } & \\
\hline & $\mathrm{x}_{1}$ & $\mathrm{x}_{2}$ & $\xi_{1}$ & $\xi_{2}$ & \\
\hline Origen & 0 & 0 & 40 & 835 & - \\
\hline$\Delta$ & 0,25 & 0,1968 & 2,5 & 3 & - \\
\hline Orig. $+\Delta$ & 0,25 & 0,1968 & 42,5 & 838 & 268,8353 \\
\hline Orig. $+2 \Delta$ & 0,5 & 0,3936 & 45 & 841 & 269,9515 \\
\hline Orig. $+3 \Delta$ & 0,75 & 0,5904 & 47,5 & 844 & 272,1383 \\
\hline Orig. $+4 \Delta$ & 1 & 0,7872 & 50 & 847 & 268,9122 \\
\hline Orig. $+5 \Delta$ & 1,25 & 0,984 & 52,5 & 850 & 266,7368 \\
\hline
\end{tabular}

Elaboración propia

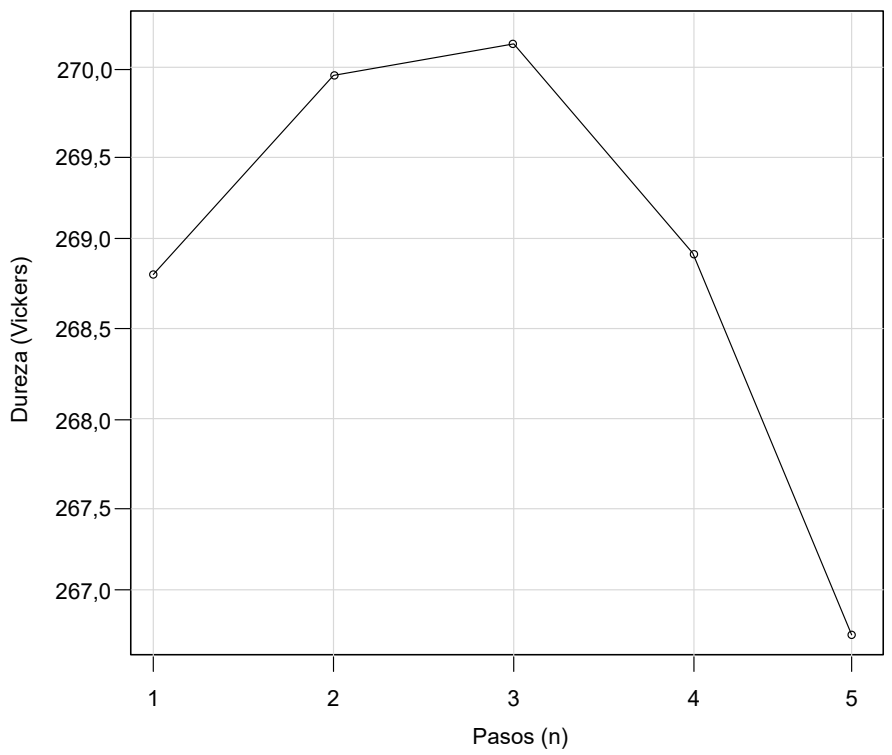

Figura 6. Gráfica de la dureza contra los pasos de la trayectoria de ascenso más pronunciado

Elaboración propia 


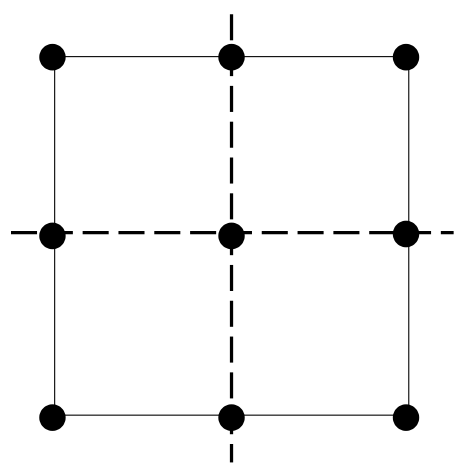

Figura 7. Esquema de los puntos axiales agregados $\operatorname{con} \alpha=1$

Elaboración propia

En la tabla 9 se presentan las mediciones obtenidas y en la tabla 10 los resultados del análisis de varianza. La normalidad de los residuos fue verificada a través de un QQ-Plot, la independencia de los residuos fue verificada a través de un gráfico de tendencia.

Tabla 9

Diseño factorial $3^{2}$ con base en la trayectoria de experimentación

\begin{tabular}{|c|c|c|c|c|c|}
\hline \multirow{3}{*}{ Prueba } & \multicolumn{4}{|c|}{ Factores del proceso } & \multirow{3}{*}{$\begin{array}{c}\begin{array}{c}\text { Variable de } \\
\text { respuesta }\end{array} \\
\text { Dureza } \\
\text { (Vickers) }\end{array}$} \\
\hline & \multicolumn{2}{|c|}{ Variables naturales } & \multicolumn{2}{|c|}{ Variables codificadas } & \\
\hline & $\begin{array}{l}\text { Tiempo de } \\
\text { austenizado } \\
\text { (minutos) }\end{array}$ & $\begin{array}{c}\text { Temperatura de } \\
\text { austenizado } \\
\left({ }^{\circ} \mathrm{C}\right)\end{array}$ & $\begin{array}{l}\text { Tiempo de } \\
\text { austenizado } \\
\text { (minutos) }\end{array}$ & $\begin{array}{c}\text { Temperatura de } \\
\text { austenizado } \\
\left({ }^{\circ} \mathrm{C}\right)\end{array}$ & \\
\hline 1 & 45 & 841 & -1 & -1 & 269,3354 \\
\hline 2 & 47,5 & 841 & 0 & -1 & 269,2105 \\
\hline 3 & 50 & 841 & 1 & -1 & 268,1808 \\
\hline 4 & 45 & 844 & -1 & 0 & 272,0578 \\
\hline 5 & 47,5 & 844 & 0 & 0 & 271,7229 \\
\hline 6 & 50 & 844 & 1 & 0 & 268,6224 \\
\hline 7 & 45 & 847 & -1 & 1 & 269,6854 \\
\hline 8 & 47,5 & 847 & 0 & 1 & 270,2398 \\
\hline 9 & 50 & 847 & 1 & 1 & 268,9846 \\
\hline
\end{tabular}

Elaboración propia 
Tabla 10

Resultados de la regresión de segundo orden según la trayectoria de experimentación

\begin{tabular}{lcc}
\hline $\begin{array}{c}\text { Variable de } \\
\text { respuesta }\end{array}$ & Factor & $\operatorname{Pr}(>|\mathrm{t}|)$ \\
\hline Dureza & Intercepto & 0,0056 \\
& Tiempo de austenizado & 0,3798 \\
& Temperatura de austenizado & 0,0057 \\
& Tiempo de austenizado & 0,0017 \\
& Temperatura de austenizado 2 & 0,0057 \\
& Tiempo de austenizado, temperatura de austenizado & 0,3669 \\
\hline
\end{tabular}

Elaboración propia

En la ecuación de regresión, para garantizar la jerarquía se decidió conservar el término lineal asociado al tiempo de austenizado. La ecuación de regresión de segundo orden está dada por:

$$
y=-609648+29.76 x_{1}+476.7 x_{2}-0.6173 x_{1}^{2}-0.2826 x_{2}^{2}
$$

De igual manera, se presenta la gráfica de la superficie de respuesta (figura 8).

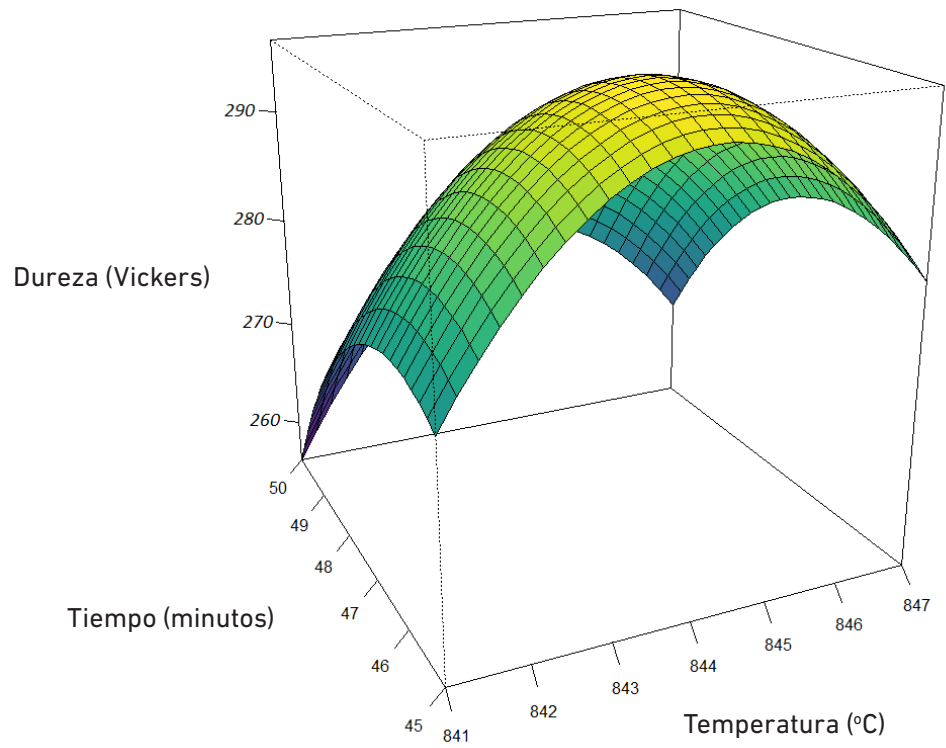

Figura 8. Superficie de respuesta

Elaboración propia 
A partir de la regresión, la cual obtuvo un $p$-value igual a 0,0007 y un valor $R^{2}$ ajustado correspondiente a 0,9903 , se determinó la ecuación polinómica que permite establecer la relación entre la dureza, la temperatura y tiempo de austenizado. De acuerdo con esto y teniendo como referencia las restricciones del proceso para ambos factores, se determinó que, con un tiempo de 48,61 minutos y $846,49{ }^{\circ} \mathrm{C}$, se obtiene la máxima dureza referente a 284,69 Vickers.

Con el fin de constatar de manera práctica este resultado, se realizó el proceso de temple a tres probetas bajo un tiempo de 48,61 minutos y $846,49{ }^{\circ} \mathrm{C}$ debido a la resolución del horno (tabla 11).

Tabla 11

Resultados del experimento confirmatorio

\begin{tabular}{ccccc}
\hline & \multicolumn{2}{c}{ Factores del proceso } & Variable de respuesta & $\begin{array}{c}\text { Predicción del modelo } \\
\text { de regresión }\end{array}$ \\
\cline { 2 - 5 } Probeta & $\begin{array}{c}\text { Tiempo de } \\
\text { austenizado } \\
\text { [minutos }]\end{array}$ & $\begin{array}{c}\text { Temperatura de } \\
\text { austenizado } \\
\left({ }^{\circ} \mathrm{C}\right)\end{array}$ & $\begin{array}{c}\text { Dureza } \\
\text { (Vickers) }\end{array}$ & $\begin{array}{c}\text { Dureza } \\
\text { (Vickers) }\end{array}$ \\
\hline 1 & 48,61 & 846,0 & 285,3900 & 276,587 \\
2 & 48,61 & 846,0 & 284,2968 & 276,587 \\
3 & 48,61 & 846,0 & 285,1922 & 276,587 \\
\hline
\end{tabular}

Elaboración propia

Con base en los resultados obtenidos en el experimento confirmatorio y teniendo como referencia una media de dureza de 284,9597 Vickers, se infiere que el punto de inflexión que maximiza la dureza fue hallado satisfactoriamente.

\section{CONCLUSIONES Y RECOMENDACIONES}

Según el experimento realizado, la dureza sí tiene relación con el factor temperatura y tiempo de austenizado, mientras que la rugosidad no. Con estos resultados se ajustó una superficie de respuesta para la dureza en función de estas dos variables, encontrando que la máxima dureza estimada se consigue al momento de usar un tiempo de austenizado de 48,61 minutos y una temperatura de $846{ }^{\circ} \mathrm{C}$, logrando una dureza promedio de 284,95 Vickers. A un tiempo mayor la dureza disminuye, haciendo que el material sea débil y ya no funcione de manera adecuada porque se fractura con mayor facilidad.

Adicionalmente, por la diferencia que se presenta entre la predicción de la dureza y el valor observado en el experimento confirmatorio, se recomienda agregar más puntos 
axiales que permitan estimar otro tipo de curvatura que puede ajustarse mejor a la naturaleza de la dureza.

La metodología de AHP permitió analizar de manera conjunta las dos variables de respuesta. Sin embargo, al no ser significativa, no pudo usarse para desarrollar la metodología de superficie de respuesta. Para futuras investigaciones se recomienda probar otras variables adicionales que puedan tener relación con la dureza y la rugosidad.

\section{REFERENCIAS}

Aouici, H., Bouchelaghem, H., Yallese, M., Elbah, M. y Fnides, B. (2014). Machinability investigation in hard turning of AISI D3 cold work steel with ceramic tool using response surface methodology. International Journal of Advanced Manufacturing Technology 73(9-12), pp. 1775-1788.

Ariza, E. A., Martorano, M. A. y Tschiptschin, A. P. (2015). Esfuerzos residuales generados en el tratamiento térmico de temple para un cilindro de acero de medio carbono, simulación por elementos finitos y verificación experimental. La Revista Latinoamericana de Metalurgia y Materiales 35(1), pp. 55-64.

Artigas, A., Colás, R., Toledo, S., Barra, F., Pizarro, F., Carvajal, L. y Monsalve, A. (2012). Producción en laboratorio de acero dual phase laminado en caliente, para la fabricación de cilindros de gas licuado. La Revista Latinoamericana de Metalurgia y Materiales 32(2), pp. 202-209.

Awad, O. I., Mamat, R., Ali, O. M., Azmi, W. H., Kadirgama, K., Yusri, I. M., ... Yusaf, T. (2017). Response surface methodology (RSM) based multi-objective optimization of fuel oil -gasoline blends at different water content in SI engine. Energy Conversion and Management 150, pp. 222-241.

Bagaber, S.A. y Yusoff, A. R. (2017). Multi-objective optimization of cutting parameters to minimize power consumption in dry turning of stainless steel 316 . Journal of Cleaner Production 157, pp. 30-46.

Bagaber, S. A. y Yusoff, A. R. (2018). Sustainable Optimization of Dry Turning of Stainless Steel based on Energy Consumption and Machining Cost. Procedia CIRP 77, pp. 397-400.

Caballero, S.N. y Figueroa, C. G. (1999). Medios de enfriamiento para el temple. Ingeniería Mecánica 2(2), pp. 63-66.

Costa, N. R., Lourenço, J. y Pereira, Z. L. (2011). Desirability function approach: A review and performance evaluation in adverse conditions. Chemometrics and Intelligent Laboratory Systems 107, pp. 234-244. 
De Almeida, F. A., Gomes, G. F., De Paula, V. R., Corrêa, J. E., De Paiva, A. P., De Freitas, J. H. y Turrioni, J. B. (2018). A Weighted Mean Square Error Approach to the Robust Optimization of the Surface Roughness in an AISI 12L14 Free-Machining SteelTurning Process. Journal of Mechanical Engineering 64(3), pp. 147-156.

D'Mello, G. D. y Pai, P. S. (2018). Surface roughness modeling in high speed turning of ti-6Al-4V using response surface methodology. Materials Today: Proceedings 5(5), pp. 11686-11696. doi: 10.1016/j.matpr.2018.02.138

D'Mello, G., Pai, P. S. y Puneet, N. P. (2018). Surface Roughness Prediction in High Speed Turning of Ti6Al-4V: A Comparison of Techniques. Materials Science and Engineering 376, pp. 1-10. doi: 10.1088/1757-899X/376/1/012115

Franco, F. y Harrinson Paz, J. (2004). Tratamiento Térmico de Aceros de Bajo Carbono en Horno de Atmósfera Controlada. Ingeniería y Competitividad 6(2), pp. 56-63.

Gómez Barrantes, C. F. y Romero Pinzón, H. F. (2017). Optimización de la rugosidad superficial en acero AISI 1045 utilizando el método de superficie de respuesta. (Tesis de pregrado). Bogotá: Universidad Distrital Francisco José de Caldas.

González Guevara, J. M., Manrique Torres, M. R. y Quiroga Sanchez, A. M. (2016). Diseño de un proceso de tratamiento térmico para el mejoramiento de las propiedades mecánicas de una herramienta de acero de conformado en frío. (Tesis de pregrado). Bogotá: Pontificia Universidad Javeriana.

Gordillo, M., Rodríguez, E. D. y de Gutiérrez, R. M. (2014). Optimización de la resistencia mecánica de cementos binarios MK/GBFS activados alcalinamente por Metodología de Superficie de Respuesta. Ingeniería y Ciencia 10(19), pp. 197-220.

Gresesqui-Lobaina, E., Rodríguez-González, I. y Fernández-Columbié, T. (2017). Caracterización del acero 70XL empleado en la fabricación de bolas para la molienda del Clinker. Minería y Geología 33(4), pp. 486-497.

Kalpakjian, S. y Schmid, S. (2008). Manufactura, ingeniería y tecnología. México: Pearson Educación.

Koohestanian, E., Samimi, A., Mohebbi-Kalhori, D. y Sadeghi, J. (2017). Sensitivity analysis and multi-objective optimization of $\mathrm{CO} 2 \mathrm{CPU}$ process using response surface methodology. Energy 122, pp. 570-578. doi: 10.1016/j.energy.2017.01.129

Lario, J., Castaño, O., Viera, M., Segovia, F. y Amigo, V. (2018). Efecto de un tratamiento térmico sobre la composición química y morfología de nanotubos de Ti02 obtenidos por anodizado. La Revista Latinoamericana de Metalurgia y Materiales 38(1), pp. 100-109.

Lazcano-Ugalde, E. M., Méndez-Macías, G. y Medina-Mendoza, J. (2016) Análisis de falla de pernos de acero AISI 12L14. Revista de Aplicaciones de la Ingeniería 3(6), pp. 1-7. 
Mohammed, B. S., Achara, B. E. y Liew, M. S. (2018). The influence of high temperature on microstructural damage and residual properties of nano-silica-modified (NS-modified) self-consolidating engineering cementitious composites (SC-ECC) using response surface methodology (RSM). Construction and Building Materials 192, pp. 450-466.

Morales, I., Hormaza, W. y Méndez, L. (2009). Efecto del tratamiento térmico de desestabilización en el desgaste de hierros blancos de alto cromo. Revista de Ingeniería 30, pp. 41-48.

Morales Tamayo, Y., Pérez Rodríguez, R., Zambrano Robledo, P., Ávila Rondón, R. y Zamora Hernández, Y. (2014). Estudio experimental de la rugosidad superficial en el torneado en seco de alta velocidad del acero AISI 316L destinado a la agroindustria alimenticia. Revista Ciencias Técnicas Agropecuarias 23(3), pp. 54-59.

Morales Villamil, D. A. (2009). Determinación de parámetros para la operación de cilindrado de acero SAE12L 14, bronce SAE40 y aluminio SAE 1060 en el torno PC Turn 125 por medio de una metodología experimental. (Tesis de pregrado). Bogotá: Pontificia Universidad Javeriana.

Nekouei, R., Akhaghi, R., Ravanbakhsh, A., Tahmasebi, R., Moghaddam, A. y Mahrouei, M. (2016). A Study of the Effect of Two-Stage Tempering on Mechanical Properties of Steel 30CrMnSi Using Analysis on Response Surface in Design of Experiment. Metal Science \& Heat Treatment 57(11-12), pp. 694-701.

Paredes, Y. (2016). Uso de la metodología de superficie de respuesta en la definición de parámetros que permitan mejorar la dureza del acero 12L14 a través de cementado. (Tesis de pregrado). Bogotá: Pontificia Universidad Javeriana.

Parida, A. K., Routara, B. C. y Bhuyan, R. K. (2015). Surface roughness model and parametric optimization in machining of GFRP composite: Taguchi and Response surface methodology approach. Materials Today: Proceedings 2(4-5), pp. 3065-3074.

Pérez Ruiz, E. A., Galeano Perilla, A.C. y Negrín Hernández, L.L (2016). Evaluación de la dureza y microestructura del acero 1045 templado en sustancias refrigerantes no tradicionales. Scientia et Technica 21(3), pp. 213-218.

Pérez, W., Olaya, J. J. y Arenas, J. A. (2013). Influencia de las condiciones del tratamiento térmico sobre las propiedades mecánicas del acero $5160 \mathrm{H}$. Revista Técnica de la Facultad de Ingeniería Universidad del Zulia 36(1), pp. 23-31.

Posada Ramírez, J. E. (2009). Evaluación de la integridad superficial del acero AISI-12L14 para maquinaria, sometido a procesos de maquinado. (Tesis doctoral). Bogotá: Universidad Nacional de Colombia. 
Raissi, S. y Eslami Farsani, R. (2009). Statistical Process Optimization Through MultiResponse Surface Methodology. International Scholarly and Scientific Research \& Innovation 3(3), pp. 197-201.

Robledo, D. M. y Cárdenas, A. O. (2011). Evaluación de los parámetros de temple y revenido para el acero AISI/SAE1045 a escala industrial. Scientia et Technica 3(49), pp. 25-29.

Rodríguez-Baracaldo, R., Arroyo-Osorio, J. M. y Parra-Rodríguez, Y. (2016). Influencia del proceso de revenido en el comportamiento mecánico de un acero de fase dual de uso industrial automotriz. INGENIARE, Revista Chilena de Ingeniería 24(1), pp. 94-101.

Saaty, T.L. (2008). Decision making with the analytic hierarchy process. International Journal of Services Sciences 1(1), pp. 83-98.

Saba, F. y Raygan, S. (2017). Application of response surface methodology for modelling of TiC coating on AISI D2 steel using a mechanical milling technique. Powder Metallurgy 60(4), pp. 280-292.

Sáenz, P. L. A., Hilders, O. A., Hergueta, R. D. y Zabala, E. A. (2007). Estudio de un acero inoxidable dúplex saf 2507 sometido a un tratamiento térmico de envejecimiento a una temperatura de $850^{\circ} \mathrm{C}$ por tiempos entre 1 hora y 48 horas. Revista de la Facultad de Ingeniería Universidad Central de Venezuela 3, p. 21.

Senthilkumar, N., Tamizharasan, T. y Gobikannan, S. (2014). Application of Response Surface Methodology and Firefly Algorithm for Optimizing Multiple Responses in Turning AISI 1045 Steel. Arabian Journal for Science \& Engineering 39(11), pp. 8015-8030.

Serra, J. C., Bernasconi, G., Lagrutta, J. M., Bergesio, A., Negreira, A. y Mendoza, S. M. (2017). Evaluación De Tratamientos Térmicos en Aceros Comerciales Aisi 304L Y Aisi 316L Para Implantes Óseos. Revista Iberoamericana de Ingeniería Mecánica 21(1), pp. 23-30.

Silva Urueña, J. y Castañeda Vargas, J. F. (2015). Influencia del Tiempo de Revenido en las Propiedades Mecánicas de Dureza y Resistencia a la Tensión de un Acero SAE 1045. (Tesis de pregrado). Bogotá: Universidad Distrital Francisco José De Caldas.

Soria-Aguilar, M. J., Reyes-Guzmán, F., Carrillo-Pedroza, F. R., García-Garza, F., ÁlvarezJiménez, H. y Silva-Guajardo, L. A. (2015). Efecto del tratamiento térmico sobre las propiedades mecánicas y microestructura de un acero para tubería API 5CT J55. Ingeniería, Investigación y Tecnología 16(4), pp. 539-550. 
Srivastava, M., Maheshwari, S., Kundra, T. y Rathee, S. (2017). Multi-Response Optimization of Fused Deposition Modelling Process Parameters of ABS Using Response Surface Methodology (RSM)-Based Desirability Analysis. Materials Today: Proceedings 4(2), pp. 1972-1977.

Yaguas, 0. J. (2017). Metodología de superficie de respuesta para la optimización de una producción agrícola. Ingeniería Industrial 16(2), pp. 205-222.

Ye, W., Yang, P. y Liu, Y. (2018). Multi-objective thermodynamic optimization of a free piston Stirling engine using response surface methodology. Energy Conversion and Management 176, pp. 147-163. 
